\title{
Perfil metabolómico de resistencia a la insulina en mujeres no diabéticas con lupus eritematoso sistémico
}

\author{
Claudia Mendoza-Pinto ${ }^{1,2}$, Mario García-Carrasco,2*, Socorro Méndez-Martínez³,

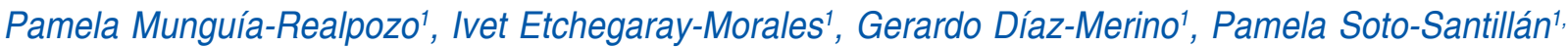 \\ Marco A. Escamilla-Márquez y Alejandro Ruiz-Arguelles ${ }^{5}$ \\ 'Unidad de Investigación de Enfermedades Autoinmunes Sistémicas, Unidad Médica de Alta Especialidad, Centro Médico Nacional Manuel Ávila \\ Camacho-CIBIOR, Instituto Mexicano del Seguro Social; ${ }^{2}$ Departamento de Inmunología y Reumatología, Facultad de Medicina, Benemérita \\ Universidad Autónoma de Puebla; ${ }^{3}$ Coordinación de Investigación, Instituto Mexicano del Seguro Social; ${ }^{4}$ Departamento de Endocrinología Unidad \\ Médica de Alta Especialidad, Centro Médico Nacional Manuel Ávila Camacho, Instituto Mexicano del Seguro Social; ${ }^{5}$ Laboratorios Clínicos de \\ Puebla. Puebla, México
}

\section{Resumen}

Introducción: En lupus eritematoso sistémico (LES) es más frecuente la prevalencia de resistencia a la insulina (RI) y síndrome metabólico (SMet) que en controles. Objetivo: Evaluar la RI en mujeres no diabéticas con LES mediante biomarcadores usando técnicas metabolómicas de alta resolución. Método: Estudio transversal en pacientes con LES. Se empleó un abordaje metabolómico usando cromatografía de líquidos de ultra-alta resolución con espectrometría de masa de alta resolución. El SMet fue evaluado de acuerdo con los criterios NCEP-ATP III. Resultados: Se incluyeron 70 pacientes con LES. Tuvieron RI y SMet 45 (64.2\%) y 27 (38.5\%), respectivamente. Pacientes con RI tenían un mayor índice de masa corporal e hipertensión con mayor frecuencia que aquellas sin RI. El daño crónico y la actividad de la enfermedad no se relacionaron con RI. Un puntaje de Quantose RI $\geq 63$ fue más elevado en pacientes con SMet (81.5 vs 53.5\%; $p=0.02)$. El puntaje Quantose $R I$ también se correlacionó con el número de criterios para SMet ( $r: 0.35 ; p=0.003)$. Conclusiones: En mujeres con LES no diabéticas, la prevalencia de RI basada en el puntaje de Quantose RI fue del 64.2\%.

PALABRAS CLAVE: Lupus eritematoso sistémico. Resistencia a la insulina. Metabolómica.

\section{Insulin resistance metabolomic profile in non-diabetic women with systemic lupus erythematosus}

\section{Abstract}

Introduction: Patients with systemic lupus erythematosus (SLE) have a higher prevalence of insulin resistance (IR) and metabolic syndrome (MetS) than controls. Objective: To evaluate IR in non-diabetic women with SLE by means of biomarkers using high-throughput metabolomic techniques. Methods: Cross-sectional study in patients with SLE. A metabolomic approach was employed using ultra-high performance liquid chromatography coupled with high-resolution mass spectrometry. MetS was evaluated according to NCEP-ATP III criteria. Results: Seventy patients with SLE were included, out of whom 45 (64.2\%) and 27 (38.5\%) had IR and MetS, respectively. Patients with IR had a higher body mass index and hypertension more often than those without IR. Chronic damage and disease activity were not related to IR. A Quantose-IR score $\geq 63$ was more common in patients with MetS (81.5 vs. $53.5 \% ; p=0.02)$. Quantose-IR score was also correlated with the number of criteria for MetS ( $r: 0.35 ; p=$ 0.003). Conclusions: In non-diabetic women with SLE, the prevalence of IR based on Quantose-IR score was $64.2 \%$.

KEY WORDS: Systemic lupus erythematosus. Insulin resistance. Metabolomics. 


\section{Introducción}

Recientemente, se ha destacado una posible asociación entre el lupus eritematoso sistémico (LES) e índices altos de resistencia a la insulina (RI) medidos por el índice de resistencia a la insulina HOMA-IR

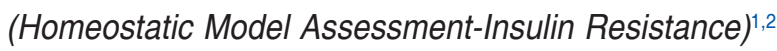
y síndrome metabólico (SMet) ${ }^{3}$. La RI, definida como una sensibilidad o receptividad disminuida de los tejidos sensibles a la insulina a esta ${ }^{4}$, ha demostrado ser un factor de riesgo independiente para diabetes mellitus tipo $2(\mathrm{DM} 2)^{5}$ y puede estar presente por años antes de la aparición de algún cambio en el control glucémico. El índice de HOMA-IR es un modelo simple que requiere una sola muestra de sangre, pero no está claro si ofrece ventajas sobre la insulina en ayuno ${ }^{6}$. Por lo tanto, existe una necesidad de un método simple para medir RI que pueda ser usado para tamizaje sistemático, estudios prospectivos, evaluación de riesgo, monitoreo terapéutico y que sea costo-efectivo para un seguimiento adecuado. La metabolómica es una tecnología analítica emergente que cuantifica de manera simultánea múltiples metabolitos en biofluidos e identifica nuevos biomarcadores que son altamente discriminatorios para alteraciones biológicas ${ }^{7}$ La cromatografía de líquidos de ultra-alta resolución con espectrometría de masas (UHPLC-HRMS) se considera una buena herramienta para la identificación de metabolitos y estudio metabolómico, especialmente para la identificación metabólica indeterminada a gran escala, debido a su eficiencia de separación ${ }^{8,9}$.

Aunque la metabolómica ha demostrado potencial en el diagnóstico de $\operatorname{LES}^{10}$ y nefritis lúpica ${ }^{11}$, la metabolómica sérica aún no ha sido estudiada para identificar las alteraciones metabólicas asociadas con la RI en estos pacientes. Por lo tanto, el objetivo de este estudio es estudiar la RI en mujeres con LES no diabéticas usando una técnica metabolómica de alto rendimiento, así como evaluar la relación entre factores de riesgo tradicional y de LES con la presencia de RI.

\section{Métodos}

En este estudio transversal con aprobación del Comité de Ética Local se incluyeron pacientes adultos con LES por los criterios del American College of Rheumatology $(A C R)^{12}$ sin eventos cardiovasculares (ECV). Se excluyeron pacientes con DM, ECV, obesidad o embarazadas. Los pacientes fueron evaluados para factores de riesgo cardiovascular y terapéutica actual (glucocorticoides actual y en los últimos seis meses), antropometría y presión arterial. La actividad del LES y daño crónico se midieron con el índice SLEDAI-2K (SLE Disease Activity Index 2000)13 y el índice SLICC (Systemic Lupus International Collaborating Clinics)/ $\mathrm{ACR}^{14}$, respectivamente. El SMet se determinó utilizando los criterios del NCEP-ATP III (National Cholesterol Education Program- Adult Treatment Panel III)15.

La UHPLC-HRMS utiliza la técnica de dilución de isótopos estables para cuantificar el ácido hidroxibutírico (AHB), I-linoleoil-glicerofosfocolina (LGPC) y el oleato (sistema Waters Acquity UHPLC, Waters Corporation, Milford, MA); el AHB, el LGPC y el ácido oleico se eluyeron con un gradiente en una columna de Waters Acquity single RP C-18 $(2.1 \mathrm{~mm} \times 50 \mathrm{~mm}$, tamaño de partícula de $1.7 \mathrm{~mm}$ ) a un caudal de $0.8 \mathrm{ml} / \mathrm{min}$ a $40^{\circ} \mathrm{C}$. La ionización se logró mediante una fuente de ionización por electroespray calentada. La cuantificación se basó en las relaciones de área de la sustancia y picos estándar internos utilizando un análisis de regresión lineal de mínimos cuadrados ponderado, generado a partir de estándares de calibración fortificados en una matriz artificial, preparado antes de cada serie analítica. La insulina plasmática se cuantificó utilizando el inmunoensayo de micropartículas quimioluminiscentes Abbott (Architect). Las concentraciones de las sustancias se utilizaron junto con un algoritmo para calcular el nivel de RI con la puntuación Quantose $\mathrm{IR}^{\mathrm{TM}}$. El valor de corte $\mathrm{RI}$ de 63 se definió por el tercil superior de las puntuaciones del estudio Europeo ${ }^{16}$.

Para el análisis estadístico, las variables cuantitativas se evaluaron con prueba t de Student o prueba $\mathrm{U}$ de Mann-Whitney y las categóricas por medio de prueba de $\chi^{2}$. Se realizó correlación de Spearman para evaluar la relación entre el número de criterios para SMet y puntaje de Quantose RI. Se utilizó el programa estadístico SPSS para Mac versión 25.0 (SPSS Inc., Chicago, IL, EE.UU.).

\section{Resultados}

Se incluyeron 70 pacientes con LES con una edad de $39.3 \pm 10.6$ años. La tabla 1 muestra los datos sociodemográficos y características relacionadas con la enfermedad. Cuarenta y cinco de los 70 pacientes (64.2\%) con LES tenían RI con una mediana de la puntuación de Quantose IR de 69 (intervalo intercuartílico [RIQ]: 52.7-80.0).

La prevalencia de RI fue mayor en pacientes con SMet en comparación con aquellos sin los criterios de NCEP-ATP III (81.5 vs. $53.5 \%$; $p=0.02$ ). 
Tabla 1. Características de los 70 pacientes con lupus eritematoso sistémico

\begin{tabular}{|c|c|}
\hline & Medición \\
\hline $\begin{array}{l}\text { Edad, años } \\
\quad \text { Media (DE) }\end{array}$ & $39.3 \pm 10.6$ \\
\hline $\begin{array}{l}\text { Duración de la enfermedad, años } \\
\text { Mediana (RIQ) }\end{array}$ & $11(8-14)$ \\
\hline $\begin{array}{l}\text { IMC, } \mathrm{kg} / \mathrm{m}^{2} \\
\text { Media (DE) }\end{array}$ & $26.3 \pm 24.2$ \\
\hline $\begin{array}{l}\text { Circunferencia de cintura, } \mathrm{cm} \\
\text { Media (DE) }\end{array}$ & $89.5 \pm 11.5$ \\
\hline $\begin{array}{l}\text { Obesidad } \\
\text { n (\%) }\end{array}$ & $12(17.1)$ \\
\hline $\begin{array}{l}\text { Presión arterial sistólica, mmHg } \\
\text { Media (DE) }\end{array}$ & $119.5 \pm 12.2$ \\
\hline $\begin{array}{l}\text { Presión arterial diastólica, mmHg } \\
\text { Media (DE) }\end{array}$ & $76.4 \pm 9.7$ \\
\hline $\begin{array}{l}\text { Fumadores } \\
\mathrm{n}(\%)\end{array}$ & $5(7.1)$ \\
\hline $\begin{array}{l}\text { Menopausia } \\
\mathrm{n}(\%)\end{array}$ & $69(43.1)$ \\
\hline $\begin{array}{l}\text { Hipertensión } \\
\text { n (\%) }\end{array}$ & $24(24.3)$ \\
\hline $\begin{array}{l}\text { Glucosa, mg/dl } \\
\text { Media (DE) }\end{array}$ & $91 \pm 17.4$ \\
\hline $\begin{array}{l}\text { Colesterol total, mg/dl } \\
\text { Media (DE) }\end{array}$ & $191.6 \pm 37.6$ \\
\hline $\begin{array}{l}\text { Triglicéridos, mg/dl } \\
\text { Media (DE) }\end{array}$ & $162.7 \pm 111.4$ \\
\hline $\begin{array}{l}\text { Colesterol-HDL mg/dl, } \\
\text { Media (DE) }\end{array}$ & $49.0 \pm 16.4$ \\
\hline $\begin{array}{l}\text { Historia familiar de ECV, n (\%) } \\
n(\%)\end{array}$ & $27(38.6)$ \\
\hline $\begin{array}{l}\text { SLEDAI-2K, puntaje } \\
\text { Media (DE) }\end{array}$ & $0.8 \pm 1.5$ \\
\hline $\begin{array}{l}\text { Anti-ADNds, positivo } \\
\mathrm{n}(\%)\end{array}$ & $12(17.1)$ \\
\hline $\begin{array}{r}\mathrm{SDI} \geq 1 \\
\mathrm{n}(\%)\end{array}$ & $12(17.4)$ \\
\hline $\begin{array}{l}\text { Glucocorticoides, uso } \\
\mathrm{n}(\%)\end{array}$ & $64(91.4)$ \\
\hline $\begin{array}{l}\text { Dosis diaria de prednisona actual, } \mathrm{mg} / \mathrm{d} \\
\text { Media (DE) }\end{array}$ & $10.0 \pm 9.0$ \\
\hline $\begin{array}{l}\text { Dosis acumulada de glucocortidoide en } 6 \text { meses, g } \\
\text { Media (DE) }\end{array}$ & $1.8 \pm 1.5$ \\
\hline $\begin{array}{l}\text { Antipalúdicos, } n \\
n(\%)\end{array}$ & $48(68.6)$ \\
\hline $\begin{array}{l}\text { Fármacos inmunosupresión, } \mathrm{n} \\
\mathrm{n}(\%)\end{array}$ & $39(55.7)$ \\
\hline $\begin{array}{l}\text { Estatinas } \\
\mathrm{n}(\%)\end{array}$ & 18 (25.7) \\
\hline
\end{tabular}

IMC: índice de masa corporal; HDL: high density lipoprotein (lipoproteína de alta densidad): DE: desviación estándar: RIQ: intervalo intercuartílico: ECV: eventos cardiovasculares; SLEDAI-2K: SLE Disease Activity Index 2000 (indice de actividad de lupus eritematoso sistémico); ADNds: ADN bicatenario; SDI: Systemic Damage Index (índice de daño sistémico).
El número de criterios de SMet correlacionó con la puntuación de Quantose IR (r: 0.35; $p=0.003$ ). EI índice de masa corporal (IMC) y la insulina fueron mayores en pacientes con puntuaciones más altas de Quantose IR en comparación con pacientes con Quantose IR normal (Tabla 2). La hipertensión fue significativamente más frecuente en el primer grupo. No se encontraron otras diferencias significativas en otras variables sociodemográficas o clínicas. Tampoco existieron diferencias de la dosis actual ni acumulada de glucocorticoides (GCT) entre los pacientes con o $\sin \mathrm{RI}$.

\section{Discusión}

Nuestros resultados confirman que la RI es frecuente en el LES, demostrado mediante marcadores metabólicos novedosos para riesgo de diabetes. El perfil bioquímico basado en espectrometría de masas es un enfoque tecnológico emergente para identificar biomarcadores que pueden servir como marcadores metabólicos y como base de pruebas diagnósticas novedosas ${ }^{7,17,18}$, como se demuestra en un estudio previo para su utilidad para el diagnóstico de LES ${ }^{11}$. Además, estudios recientes han utilizado esta técnica para identificar biomarcadores predictivos del desarrollo futuro de la $\mathrm{DM} 2^{19}$.

Quantose RI es una prueba para la RI desarrollada y validada dentro del estudio de Relación entre Sensibilidad a la Insulina y Enfermedad Cardiovascular $(\mathrm{RISC})^{8}$. Ha demostrado utilidad en la predicción de la progresión de tolerancia normal a intolerancia a la glucosa y es superior a otras mediciones basales simples (insulina en ayuno, IMC, glucosa en ayuno, HOMA-IR) en este contexto. El puntaje final fue desarrollado para estimar el valor obtenido del clamp hiperinsulinémico-euglucémico, el estándar de oro para determinar sensibilidad a la insulina, dentro de un estudio prospectivo, observacional de una cohorte de 1,277 clínicamente sanos, sujetos no diabéticos reclutados de 13 países europeos ${ }^{20}$. Es la primera y única prueba desarrollada y validada clínicamente usando el clamp.

El presente estudio es el primero en emplear la prueba de Quantose IR en pacientes con LES. Estudios previos han encontrado que los niveles de insulina $y$ HOMA-IR aumentaron significativamente en los pacientes con LES en comparación con los controles ${ }^{21,22}$. En nuestro estudio se encontró que la puntuación de Quantose IR > 63 estaba relacionada con algunos componentes de SMet, como el IMC y la hipertensión. 
Gaceta Médica de México. 2021;157

Tabla 2. Características de pacientes con lupus eritematoso sistémico con y sin resistencia a la insulina $(\mathrm{n}=70)$

\begin{tabular}{|c|c|c|c|}
\hline & \multicolumn{2}{|c|}{ Resistencia insulina } & \multirow[t]{2}{*}{ p } \\
\hline & $\begin{array}{l}\text { Quantose }<63 \\
\quad(n=25)\end{array}$ & $\begin{array}{l}\text { Quantose } \geq 63 \\
\quad(n=45)\end{array}$ & \\
\hline Edad, años & $41.04 \pm 9.93$ & $38.42 \pm 11.04$ & 0.87 \\
\hline Duración de la enfermedad, años & $11.04 \pm 5.42$ & $11.73 \pm 7.20$ & 0.52 \\
\hline Menopausia, n (\%) & $34(43.0)$ & $35(43.2)$ & 0.55 \\
\hline Historia familiar de ECV, n (\%) & $10(40.0)$ & $17(37.8)$ & 0.52 \\
\hline Tabaquismo activo, n\% & $2(8.0)$ & $3(6.7)$ & 0.80 \\
\hline Actividad física total, horas/semana & $2.17 \pm 1.48$ & $2.79 \pm 2.30$ & 0.12 \\
\hline $\mathrm{IMC}, \mathrm{kg} / \mathrm{m}^{2}$ & $23.7(21-27)$ & $27(25-30)$ & $<0.01$ \\
\hline Circunferencia de cintura, $\mathrm{cm}$ & $85.84 \pm 10.88$ & $91.64 \pm 11.57$ & 0.89 \\
\hline Índice cintura-cadera & $0.91 \pm 0.12$ & $0.91 \pm 0.08$ & 0.24 \\
\hline Hipertensión, n (\%) & $2(8.0)$ & 15 (33.3) & 0.02 \\
\hline SLEDAI-2K, puntaje & $1.00 \pm 1.50$ & $0.82 \pm 1.52$ & 0.89 \\
\hline SLICC $\geq 1$ & $7(28.0)$ & $10(22.2)$ & 0.77 \\
\hline Anti-ADNds positivo, n (\%) & $4(16.7)$ & $8(17.8)$ & 0.59 \\
\hline $\mathrm{C} 3, \mathrm{mg} / \mathrm{dl}$ & $96.23 \pm 21.77$ & $103.74 \pm 24.36$ & 0.45 \\
\hline $\mathrm{C} 4, \mathrm{mg} / \mathrm{dl}$ & $17.56 \pm 8.42$ & $17.50 \pm 8.03$ & 0.80 \\
\hline Dosis diaria de prednisona, $\mathrm{mg}$ & $9.50 \pm 7.39$ & $10.38 \pm 9.89$ & 0.52 \\
\hline Dosis acumulada de glucocorticoide, g/6 meses & $1.96 \pm 1.75$ & $1.75 \pm 1.42$ & 0.35 \\
\hline Antipalúdicos, n (\%) & $15(60.0)$ & $33(73.3)$ & 0.29 \\
\hline Inmunosupresor, n (\%) & $9(36.0)$ & $30(66.7)$ & 0.02 \\
\hline Estatinas, n (\%) & $5(20.0)$ & $13(28.9)$ & 0.57 \\
\hline Glucosa, mg/dl & $90.70 \pm 15.95$ & $92.02 \pm 18.37$ & 0.36 \\
\hline Colesterol total, mg/dl & $192.96 \pm 36.90$ & $190.92 \pm 38.40$ & 0.75 \\
\hline Triglicéridos, mg/dl & $151.92 \pm 86.24$ & $168.73 \pm 123.75$ & 0.73 \\
\hline Niveles bajos de HDL n, (\%) & $3(12.0)$ & $14(31.1)$ & 0.08 \\
\hline Insulina, $\mu \mathrm{U} / \mathrm{I}$ & $5.78 \pm 1.82$ & $14.26 \pm 8.62$ & $<0.01$ \\
\hline
\end{tabular}

Además, Quantose IR también se correlacionó con el número de criterios de SMet, por lo tanto, esta herramienta podría ser útil en el seguimiento y los cambios derivados de modificaciones dietéticas, conductuales y farmacológicas.

Recientemente se han informado hallazgos similares en esclerosis múltiple, y podría ser útil investigar la RI en enfermedades autoinmunes crónicas, ya que puede complicar la enfermedad subyacente y deteriorar aún más la calidad de vida ${ }^{23}$.

El presente estudio tiene algunas limitaciones. Primero, las mediciones realizadas fueron realizadas en una sola ocasión, por lo tanto, una clasificación errónea de los posibles factores de riesgo podría haber afectado a nuestras estimaciones; además, la ausencia de un grupo control sano limita obtener una frecuencia 
comparativa de nuestros resultados. En segundo lugar, nuestro estudio solo fue comparativo, exploratorio, faltarían estudios con un mayor tamaño de la muestra que permite realizar un análisis multivariante controlando para factores de confusión. En tercer lugar, hay incertidumbres que podrían restringir la extrapolación de nuestros hallazgos a otros pacientes con LES con mayor actividad de la enfermedad, debido a que la mayoría de contaban una actividad leve de la enfermedad. Finalmente, el valor predictivo del índice Quantose IR sería necesario evaluarlo en estudios longitudinales de gran escala.

\section{Conclusiones}

En conclusión, en este estudio exploratorio, los pacientes con LES tuvieron un alto índice de RI según lo evaluado por el perfil metabolómico. Este es el primer estudio evaluando la RI en pacientes con LES utilizando un perfil metabolómico. Se encontró que esta $\mathrm{RI}$ en pacientes con LES es frecuente en aquellos con un mayor IMC y con hipertensión arterial.

\section{Agradecimientos}

Los autores agradecen el apoyo recibido de parte de la Vicerrectoría de Investigación y Estudios de Posgrado (VIEP) de la Benemérita Universidad Autónoma de Puebla, y al Consejo Nacional de Ciencia y Tecnología (Conacyt).

\section{Financiamiento}

Este proyecto fue financiado en parte por el Instituto Mexicano del Seguro Social con apoyo de beca para alumno de maestría.

\section{Conflicto de Intereses}

Los autores declaran no tener conflicto de intereses.

\section{Responsabilidades éticas}

Protección de personas y animales. Los autores declaran que los procedimientos seguidos se conformaron a las normas éticas del comité de experimentación humana responsable y de acuerdo con la Asociación Médica Mundial y la Declaración de Helsinki.

Confidencialidad de los datos. Los autores declaran que han seguido los protocolos de su centro de trabajo sobre la publicación de datos de pacientes.
Derecho a la privacidad y consentimiento informado. Los autores han obtenido el consentimiento informado de los pacientes y/o sujetos referidos en el artículo. Este documento obra en poder del autor de correspondencia.

\section{Bibliografía}

1. Tso TK, Huang WN. Elevation of fasting insulin and its association with cardiovascular disease risk in women with systemic lupus erythematosus. Rheumatol Int. 2009;29:735-42.

2. Kuo C-Y, Tsai TY, Huang YC. Insulin resistance and serum levels of adipokines in patients with systemic lupus erythematosus: a systematic review and meta-analysis. Lupus. 2020;29:1078-84.

3. Parker B, Urowitz MB, Gladman DD, Lunt M, Bae S-C, Sanchez-Guerrero $\mathrm{J}$, et al. Clinical associations of the metabolic syndrome in systemic lupus erythematosus: data from an international inception cohort. Ann Rheum Dis. 2013;72:1308-14.

4. Petrie JR. Evidence-based estimation of insulin resistance. Diabetologia. 2014;57:1743-5

5. Yang G, Li C, Gong Y, Fang F, Tian H, Li J, et al. Assessment of insulin resistance in subjects with normal glucose tolerance, hyperinsulinemia with normal blood glucose tolerance, impaired glucose tolerance, and newly diagnosed type 2 diabetes (Prediabetes Insulin Resistance Research). J Diabetes Res. 2016;2016:9270768.

6. Matthews DR, Hosker JP, Rudenski AS, Naylor BA, Treacher DF, Turner RC. Homeostasis model assessment: insulin resistance and beta-cell function from fasting plasma glucose and insulin concentrations in man. Diabetologia. 1985;28:412-9.

7. Nicholson JK, Lindon JC. Systems biology: Metabonomics. Nature. 2008;455:1054-6.

8. Cobb J, Gall W, Adam K-P, Nakhle P, Button E, Hathorn J, et al. A novel fasting blood test for insulin resistance and prediabetes. J Diabetes Science Technology. 2013;7:100-10.

9. Tripathy D, Cobb JE, Gall W, Adam K-P, George T, Schwenke DC, et al. A novel insulin resistance index to monitor changes in insulin sensitivity and glucose tolerance: the ACT NOW study. J Clin End Met. 2015;100:1855-62.

10. Wu T, Xie C, Han J, Ye Y, Weiel J, Li Q, et al. Metabolic disturbances associated with systemic lupus erythematosus. PloS One. 2012;7:e37210.

11. Guleria A, Pratap A, Dubey D, Rawat A, Chaurasia S, Sukesh E, et al. NMR based serum metabolomics reveals a distinctive signature in patients with Lupus Nephritis. Scientific Rep. 2016;6:35309.

12. Hochberg MC. Updating the American College of Rheumatology revised criteria for the classification of systemic lupus erythematosus. Arthritis Rheum. 1997;40:1725.

13. Gladman DD, Ibanez D, Urowitz MB. Systemic lupus erythematosus disease activity index 2000. J Rheumatol. 2002;29:288-91.

14. Gladman D, Ginzler E, Goldsmith C, Fortin P, Liang M, Urowitz M, et al. The development and initial validation of the Systemic Lupus International Collaborating Clinics/American College of Rheumatology damage index for systemic lupus erythematosus. Arthritis Rheum. 1996;39:363-9.

15. Grundy SM, Cleeman JI, Daniels SR, Donato KA, Eckel RH, Franklin BA, et al. Diagnosis and management of the metabolic syndrome: an American Heart Association/National Heart, Lung, and Blood Institute Scientific Statement. Circulation. 2005;112:2735-52.

16. Cobb J, Eckhart A, Motsinger-Reif A, Carr B, Groop L, Ferrannini E. Hydroxybutyric acid is a selective metabolite biomarker of impaired glucose tolerance. Diabetes Care. 2016;39:988-95.

17. Suhre K, Meisinger C, Doring A, Altmaier E, Belcredi P, Gieger C, et al. Metabolic footprint of diabetes: a multiplatform metabolomics study in an epidemiological setting. PloS One. 2010;5:e13953.

18. Gall WE, Beebe K, Lawton KA, Adam K-P, Mitchell MW, Nakhle PJ, et al. Alpha-hydroxybutyrate is an early biomarker of insulin resistance and glucose intolerance in a nondiabetic population. PloS One. 2010;5:e10883.

19. Ferrannini E, Natali A, Camastra S, Nannipieri M, Mari A, Adam K-P, et al. Early metabolic markers of the development of dysglycemia and type 2 diabetes and their physiological significance. Diabetes, 2013:62:1730-7.

20. Afshin A, Forouzanfar MH, Reitsma MB, Sur P, Estep K, Lee A, et al. Health effects of overweight and obesity in 195 countries over 25 years. N Engl J Med. 2017;377:13-27.

21. Lozovoy MAB, Simao ANC, Oliveira SR, Iryioda TM V, Panis C, Cecchini R, et al. Relationship between iron metabolism, oxidative stress, and insulin resistance in patients with systemic lupus erythematosus. Scand J Rheumatol. 2013:42:303-10.

22. Wang TJ, Larson MG, Vasan RS, Cheng S, Rhee EP, McCabe E, et al. Metabolite profiles and the risk of developing diabetes. Nature Med. 2011;17:448-53

23. Ruiz-Arguelles A, Mendez-Huerta MA, Lozano CD, Ruiz-Arguelles GJ. Metabolomic profile of insulin resistance in patients with multiple sclerosis is associated to the severity of the disease. Mult Scler Relat Disord. 2018;25:316-21. 